\title{
A photometric study of the young open cluster NGC $1220^{\star, \star \star}$
}

\author{
S. Ortolani ${ }^{1}$, G. Carraro ${ }^{1}$, S. Covino ${ }^{2}$, E. Bica ${ }^{3}$, and B. Barbuy ${ }^{4}$ \\ 1 Dipartimento di Astronomia, Università di Padova, Vicolo Osservatorio 2, 35122 Padova, Italy \\ e-mail: ortolani@pd.astro.it \\ 2 Osservatorio Astronomico di Milano, Italy \\ e-mail: covino@merate.mi .astro.it \\ 3 Universidade Federal do Rio Grande do Sul, Dept. de Astronomia, CP 15051, Porto Alegre 91501-970, Brazil \\ e-mail: bica@if.ufrgs.br \\ ${ }^{4}$ Universidade de Sao Paulo, Dept. de Astronomia, CP 3386, Sao Paulo 01060-970, Brazil \\ e-mail: barbuy@astro.iag.usp.br
}

Received 9 April 2002 / Accepted 27 May 2002

\begin{abstract}
We present $U B V$ CCD observations obtained in the field of the northern open cluster NGC 1220, for which little information is available. We provide also $B V$ CCD photometry of a field $5^{\prime}$ northward of NGC 1220 to take into account field star contamination. We argue that NGC 1220 is a young compact open cluster, for which we estimate a core radius in the range 1.5-2.0 arcmin. We identify 26 likely candidate members with spectral type earlier than $A 5$, down to $V_{\mathrm{o}}=15.00 \mathrm{mag}$ on the basis of the position in the two-colour Diagram and in the Colour Magnitude Diagrams (CMDs). By analyzing the distribution of these stars in the colour-colour and CMDs, we find that NGC 1220 has a reddening $E(B-V)=0.70 \pm 0.15 \mathrm{mag}$, is placed $1800 \pm 200 \mathrm{pc}$ distant from the Sun, and has an age of about 60 Myrs. The cluster turns out to be located about $120 \mathrm{pc}$ above the Galactic plane, relatively high with respect to its age.
\end{abstract}

Key words. open clusters and associations: individual: NGC 1220 - open clusters and associations: general

\section{Introduction}

NGC 1220 (Collinder 37, OCL 380) is an open cluster in Perseus for which not much information is available. Its near anticenter projection $\left(l=143.03^{\circ}, b=-3.96^{\circ} ; \mathrm{J} 2000 \alpha=\right.$ $\left.3^{\mathrm{h}} 11^{\mathrm{m}} 40^{\mathrm{s}}, \delta=53^{\circ} 20^{\prime} 45^{\prime \prime}\right)$, small angular size $\left(\approx 3^{\prime}\right)$ and its contrasted appearance with respect to the field, as inspected on DSS (Digitized Sky Survey) images, make it an interesting target. To our knowledge, this cluster remained unstudied insofar, but for the very preliminary investigation by Phelps et al. (1994), who presented $B V I$ instrumental CMDs. Since they were looking for old open cluster candidates, their analysis was limited to the remark that this cluster has to be young, since no clear giant branch and clump were detected. Therefore, these authors do not report any estimates of the cluster fundamental parameters. In the present study we provide the first calibrated $U B V C C D$ photometry of NGC 1220 , aiming at determining its basic parameters, such as reddening, distance and age, which are fundamental to understand the disk sub-system which the cluster belongs to.

Send offprint requests to: G. Carraro,

e-mail: giovanni.carraro@unipd.it

* Based on observations carried out at Observatorio de S. Pedro Martir, UNAM, Mexico.

$\star \star$ Photometry is only available in electronic form at the CDS via anonymous ftp to cdsarc.u-strasbg.fr (130.79.128.5) or via http://cdsweb.u-strasbg.fr/cgi-bin/qcat?J/A+A/391/179
The plan of this paper is as follows.

In Sect. 2 we present the observations and data reduction and in Sect. 3 we derive an estimate of the cluster diameter. In Sect. 4 we describe the CMDs, whilst in Sect. 5 we derive cluster reddening, and isolate candidate members. Section 6 is dedicated to derive NGC 1220 distance and age. Finally, in Sect. 7 we draw some conclusions and suggest further lines of research.

\section{Observations and data reduction}

Observations were carried out with the CCD SIT \#1 camera at the Observatorio Astronómico Nacional (OAN) de S. Pedro Martir, B.C., Mexico, in the photometric nights of September $16-17,2001$. This CCD samples a 4.6 .6 .'6 field in a $1 K \times 1 K$ thinned CCD. The typical seeing was around 1.7 arcsec.

The details of the observations are listed in Tables 1 and 2, where the observed fields are reported together with the exposure times, the typical seeing and the airmass from the night of September 16 and 17, respectively. The covered region is shown in Fig. 1, where a DSS ${ }^{1}$ map is presented for NGC 1220.

The data have been reduced by using the MIDAS and DAOPHOT packages. The calibration equations obtained by observing Landolt (1992) SA 92, SA 95, PG 0220+015, PG $2331+055$ and PG $0231+051$, PG 2336+004 and

\footnotetext{
${ }^{1}$ Digital Sky Survey, http://archive.eso.org/dss/dss
} 


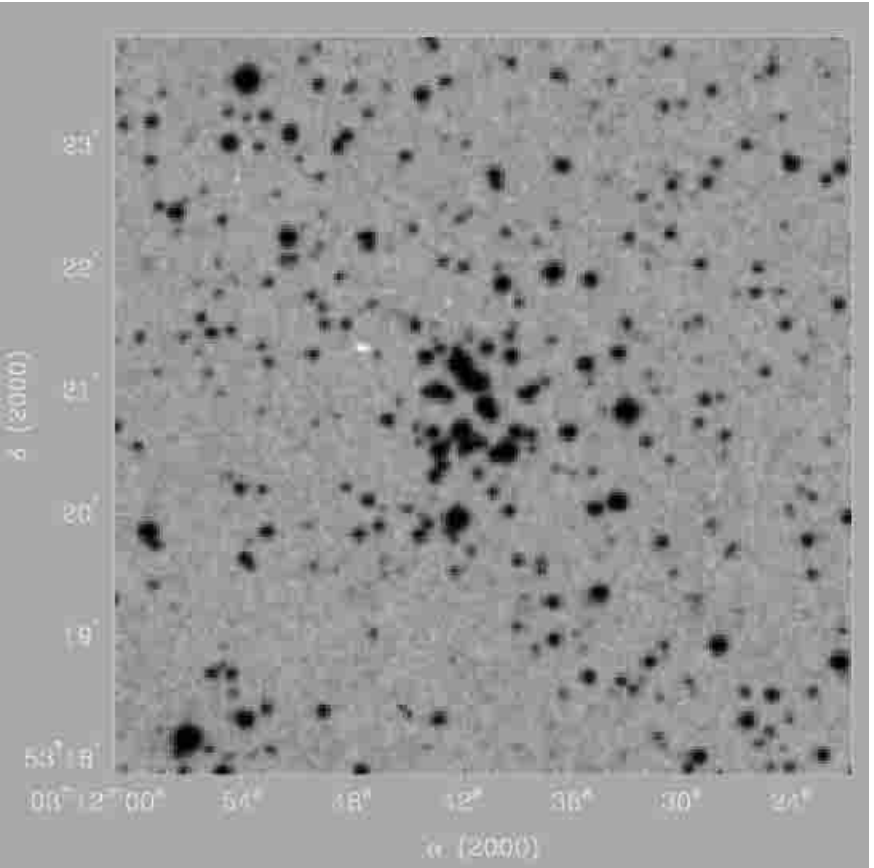

Fig. 1. A DSS red map of the covered region in the field of NGC 1220. North is up, East on the left.

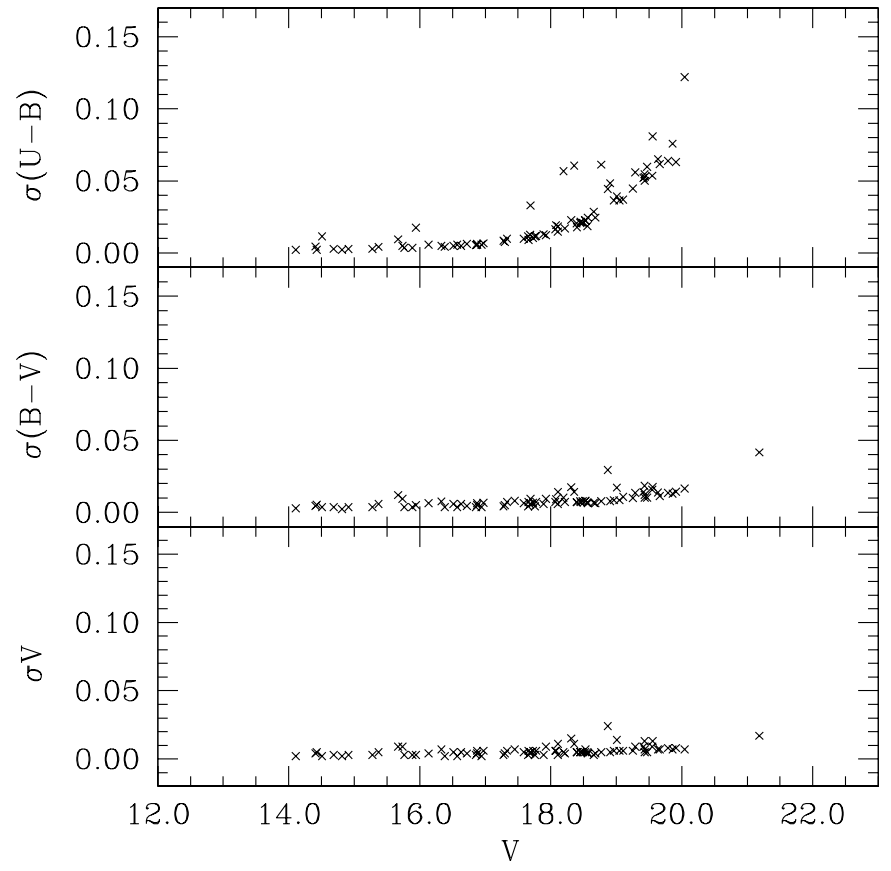

Fig. 2. Run of the magnitude and colours errors as a function of $V$ for our observations of NGC 1220.

PG 1633+099 fields along both nights for a total of 58 independent measurements, are:

$u=U+19.56 \pm 0.015+(0.04 \pm 0.02)(U-B)+0.42 X$

$b=B+21.23 \pm 0.015-(0.08 \pm 0.017)(B-V)+0.22 X$

$v=V+21.90 \pm 0.015+(0.015 \pm 0.010)(B-V)+0.14 X$

where $U B V$ are standard magnitudes, $u b v$ are the instrumental ones, and $X$ is the airmass. The zero points are for $1 \mathrm{~s}$ exposure time. The standard stars in these fields provide a very good

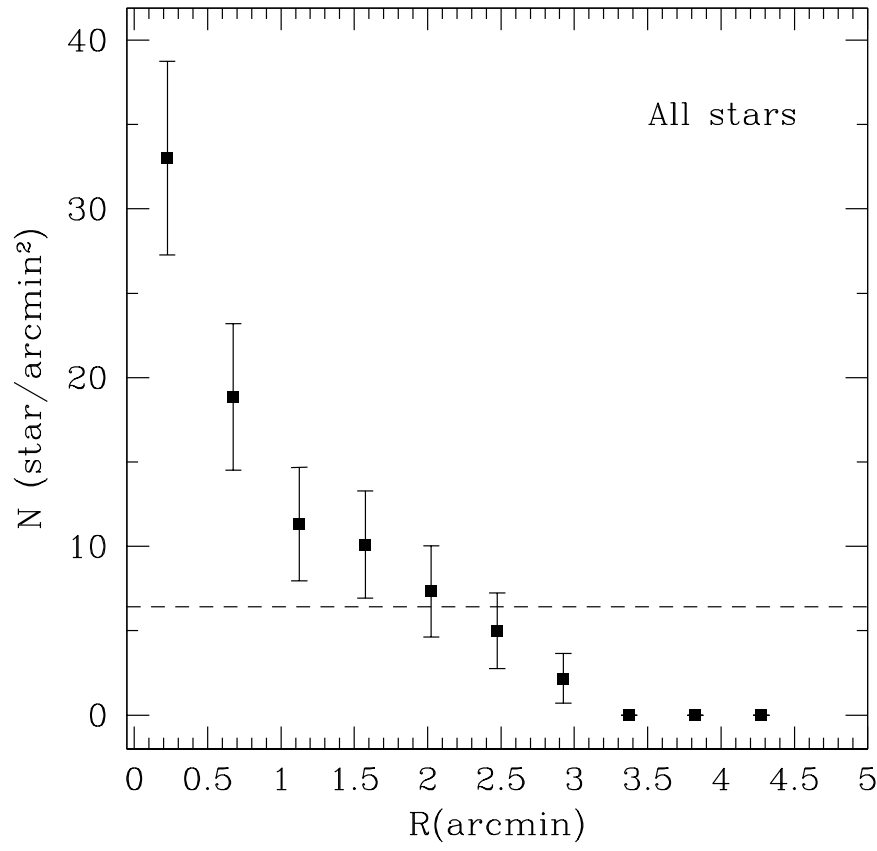

Fig. 3. Star counts in the field of of NGC 1220 as a function of radius. The dashed line indicates the background level, as derived from star counts in the accompanying field.

colour coverage from $-0.33 \leq(B-V) \leq 1.44$. Only one standard star has been excluded because of its large deviation from the average value ( $S A 95-106)$. For the extinction coefficients, we assumed the typical values of the site of $0.42,0.22$ and 0.14 fro $U, B$ and $V$ passbands, respectively, available from the OAN web site ${ }^{2}$. The photometric errors are presented in Fig. 2 for $V$, $(B-V)$ and $(U-B)$. The zero point errors of the final photometry should include also the transfer from the DAOPHOT convolved magnitudes to the wide aperture ones $(6.7$ arcsecs radius) needed to avoid any PSF and seeing variation effect. This transfer accounts for an additional \pm 0.02 mag uncertainty on all colours.

\section{Star counts and cluster size}

NGC 1220 appears as a compact cluster of about 20 stars (see Fig. 1). According to Lyngå (1987), NGC 1220 has a diameter of 2 arcmin, so our study covers the entire cluster region. To infer an estimate of the radius, we derive the surface stellar density by performing star counts in concentric rings around the center of the covered area, and then dividing by their respective surface areas. The final density profile and the corresponding Poisson error bars are depicted in Fig. 3. The dashed line in this plot represents the star counts in the accompanying field, where we estimated $6.3 \mathrm{stars} / \mathrm{arcmin}^{2}$. The cluster clearly dominates over the field up to about 2 arcmin, then it completely merges with the Galactic disk field star component. Therefore, we estimate a cluster radius of about 1.5-2.0 arcmin, somewhat larger than the value reported by Lyngå (1987).

\footnotetext{
${ }^{2}$ http://bufadora.astrosen. unam.mx
} 


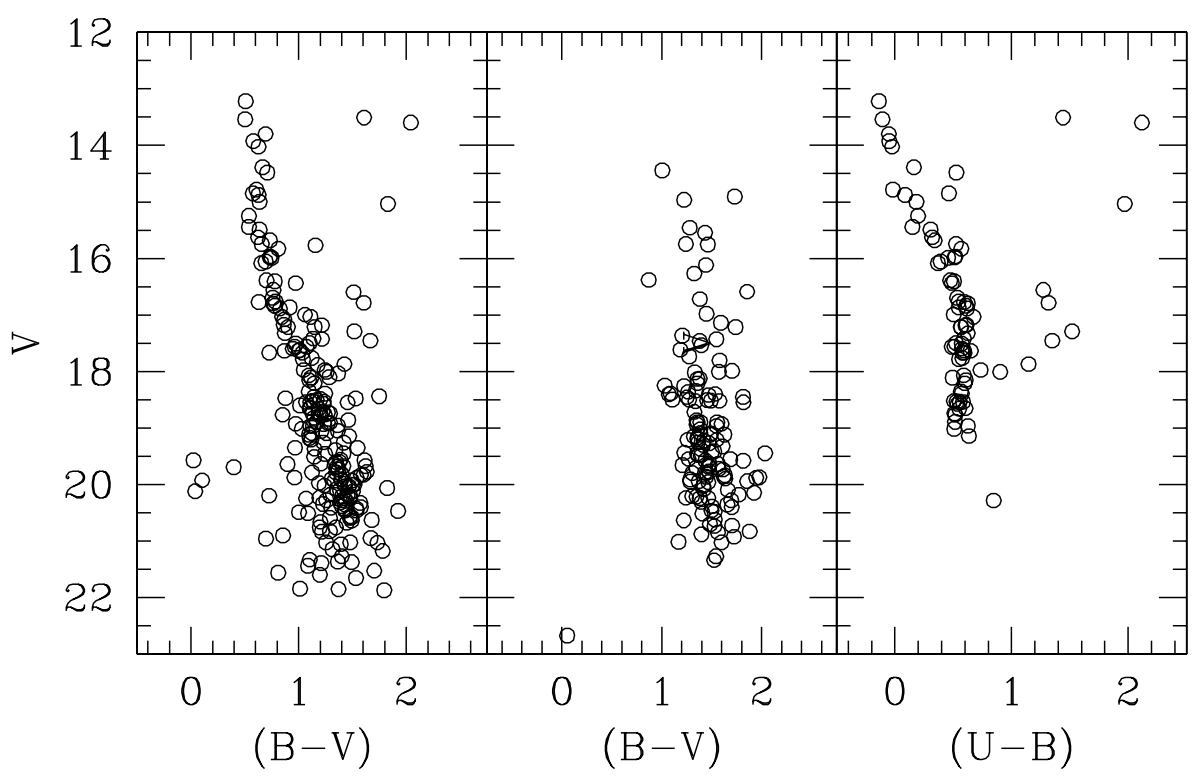

Fig. 4. CMDs of the stars in the region of NGC 1220. Left panel: all NGC 1220 stars in the $V$ vs. $(B-V)$ plane. Middle panel: all the stars in the accompanying field in the $V$ vs. $(B-V)$ plane. Right panel: all NGC 1220 stars in the $V$ vs. $(U-B)$ plane.

Table 1. Journal of observations of NGC 1220, the accompanying field, and standard star fields (September 16, 2001).

\begin{tabular}{ccccc}
\hline \hline Field & Filter & $\begin{array}{c}\text { Time integration } \\
(\mathrm{s})\end{array}$ & $\begin{array}{c}\text { Seeing } \\
\left({ }^{\prime \prime}\right)\end{array}$ & Airmass \\
\hline SA 95 & & 120 & 1.7 & 1.21 \\
& $U$ & 45 & 1.8 & 1.20 \\
& $B$ & 30,30 & 1.6 & 1.22 \\
NGC 1220 & $V$ & & & \\
& $U$ & 120 & 1.8 & 1.16 \\
& $B$ & 120,1800 & 1.9 & 1.13 \\
PG 1633+099 & $V$ & 30,900 & 1.6 & 1.14 \\
& & & & \\
& $U$ & 90 & 1.9 & 1.38 \\
PG 0231+051 & $B$ & 40 & 1.8 & 1.37 \\
& $V$ & 20 & 1.7 & 1.39 \\
& $U$ & 90 & 1.6 & 1.11 \\
& $B$ & 30,30 & 1.7 & 1.12 \\
& $V$ & $20,20,20$ & 1.7 & 1.12 \\
PG 0220+015 & & & & \\
& & & & \\
& & 90 & 1.6 & 1.12 \\
& $B$ & 30 & 1.7 & 1.12 \\
& $V$ & 20,20 & 1.8 & 1.12 \\
\hline & & & &
\end{tabular}

\section{Colour-Magnitude diagram}

The CMD for all the stars measured in the direction of NGC 1220 is shown in Fig. 4. In the left panel we plot all the stars in the $V$ vs. $(B-V)$ plane, where in the middle panel we plot in the same plane stars from the accompanying field observed $5^{\prime}$ northward of NGC 1220 . Finally, in the left panel we present the CMD in the plane $V$ vs. $(U-B)$. These CMDs are easy to interpret. On the left panel, the cluster Main Sequence (MS) extends almost vertically from $V \approx 13$ up to $V \approx 21$, although it starts widening at $V \approx 19$. This is clearly related
Table 2. Journal of observations of NGC 1220, the accompanying field, and standard star fields (September 17, 2001).

\begin{tabular}{|c|c|c|c|c|}
\hline Field & Filter & $\begin{array}{c}\text { Time integration } \\
\text { (s) }\end{array}$ & $\begin{array}{c}\text { Seeing } \\
\left({ }^{\prime \prime}\right)\end{array}$ & Airmass \\
\hline \multicolumn{5}{|l|}{ SA 92} \\
\hline & $U$ & 90 & 1.7 & 1.16 \\
\hline & $B$ & 30 & 1.8 & 1.16 \\
\hline & V & 20,20 & 1.6 & 1.16 \\
\hline \multicolumn{5}{|c|}{ NGC 1220} \\
\hline & $U$ & 1200 & 1.6 & 1.13 \\
\hline \multicolumn{5}{|c|}{ PG 2336+004 } \\
\hline & $U$ & 90 & 1.9 & 1.20 \\
\hline & $B$ & 30 & 1.8 & 1.21 \\
\hline & V & 20,20 & 1.7 & 1.21 \\
\hline \multicolumn{5}{|c|}{ PG $0231+051$} \\
\hline & $U$ & 90 & 1.6 & 1.13 \\
\hline & $B$ & 30,30 & 1.7 & 1.13 \\
\hline & V & 20,20 & 1.7 & 1.14 \\
\hline \multicolumn{5}{|l|}{ Field } \\
\hline & $B$ & 1200 & 1.6 & 1.09 \\
\hline & V & 20,600 & 1.6 & 1.10 \\
\hline \multicolumn{5}{|c|}{ PG $2331+055$} \\
\hline & $U$ & 90 & 1.6 & 1.11 \\
\hline & $B$ & 30,30 & 1.7 & 1.11 \\
\hline & $V$ & 20,20 & 1.8 & 1.14 \\
\hline
\end{tabular}

to the field star component, which is heavy in the direction of NGC 1220, a cluster located quite low in the Galactic plane. In fact (see the middle panel) the contribution of field stars is quite heavy in the magnitude interval $18 \leq V \leq 21 \mathrm{mag}$, and some stars on the red side of the MS most probably belong to the field. The appearance of this CMD suggests that we are facing a young cluster. The three very red stars in the left panel might be the signature of a possible evolved population in NGC 1220. We claim that this is very difficult. First of all, the faintest star of the triplet has a nice counterpart in the middle panel, which 


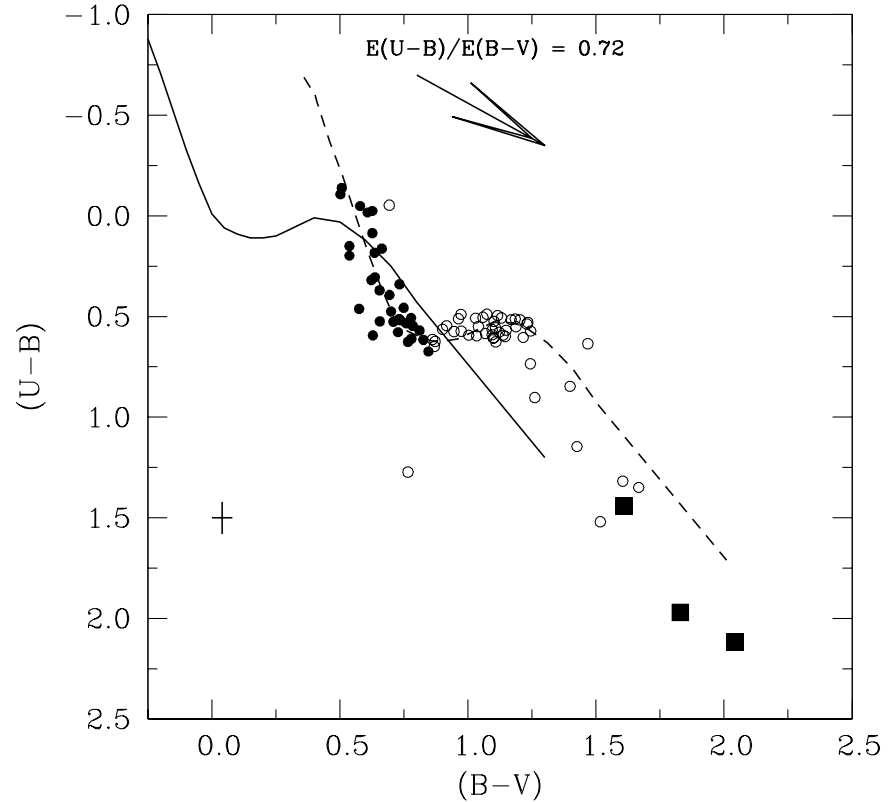

Fig. 5. Colour-colour diagram for all the stars in the field of NGC 1220 having $U B V$ photometry. The solid line is the Schmidt-Kaler (1982) empirical ZAMS, whereas the dashed line is the same ZAMS, but shifted by $E(B-V)=0.70$. Filled symbols indicate stars having reddening in the range $0.60 \leq E(B-V) \leq 0.80$, for which an unambiguous reddening solution has been possible. The filled squares indicated the three bright very red stars in Fig. 4, and the cross the typical colours error bar.

implies that it is much probably a field star. Moreover, in the case of young clusters, the $V$ vs. $(U-B)$ CMD is better suited to separate members from non members. The right panel of Fig. 4 indeed shows quite a tight MS, and some stars rightward, well detached from the MS, which presumably are all field stars.

\section{Two-colour diagram and members selection}

We derive cluster membership by grouping stars according to their mean reddening. Individual reddening values have been computed by means of the usual reddening free parameter $Q$ :

$$
Q=(U-B)-0.72 \times(B-V)
$$

and the distribution of the stars in the two colour Diagram, following the procedure outlined in detail in Carraro (2002a,b), where the young open clusters Trumpler 15 and NGC 133 have been studied, respectively. This method is a powerful one to isolate early spectral type (from $O$ to $A 5$ ) stars having common reddening, which are most probably likely cluster members (see, for a reference, the study of Trumpler 14 by Vazquez et al. 1996). Moreover the reddening based members selection nicely compares with - for instance - proper motion based members selection (see Cudworth et al. 1993; Patat \& Carraro 2001 for some clusters in the Carina region).

Our results are shown in Fig. 5, where we plot all the stars having $U B V$ photometry in the two-colour Diagram. The solid line is an empirical ZAMS from Schmidt-Kaler (1982). In this figure, we have plotted with filled symbols all the stars having a mean reddening $E(B-V)=0.70 \pm 0.15 \mathrm{mag}$ (31 stars in

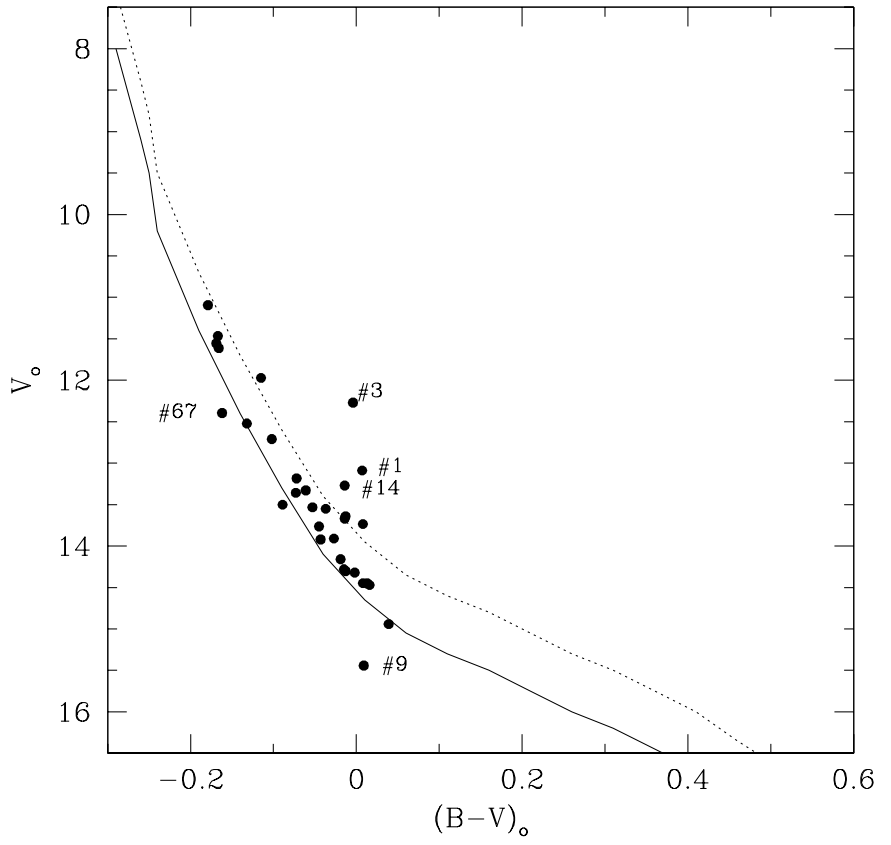

Fig. 6. Distribution of early spectral type candidate members in the reddening corrected CMD. The solid line is an empirical ZAMS shifted by $(m-M)_{V}=13.5 \pm 0.2 \mathrm{mag}$, whereas the dotted line is the same ZAMS, but 0.70 mag brighter, drawn to mimic the location of the unresolved binary stars.

total) previously determined, which obviously crowds close to a ZAMS shifted by $E(B-V)=0.70 \mathrm{mag}$ (dashed line). All these stars have common reddening and spectral type earlier than $A 5$. The spread in reddening seems to suggest that some differential reddening is present across the cluster surface. This is not unexpected, due to the cluster position in the Galactic thin disk.

The other stars are plotted with open symbols. For these latter stars an unambiguous reddening solution is not possible, since they are located in a region where larger reddening ZAMS cross the $E(B-V)=0.70$ mag ZAMS, rendering not possible to effectively disentangle members from non members. Three stars are plotted with filled squares. They are the three very red stars in Fig. 4. From their position in the twocolour diagram we argue that these stars are late spectral type ( $K$ to $M$ ) low reddening, nearby stars.

Finally, more information can be derived by considering the distribution of the stars in the reddening corrected CMD (see Fig. 6). In this figure we have plotted all the likely early spectral type members. To guide the eye, two ZAMS have been drawn. The solid one, which fits the distribution of the bulk of member stars, has been shifted by $(m-M)_{\mathrm{o}}=11.3 \pm 0.2$ (see also next section). The dotted one has been placed to mimic the expected position of presumed unresolved binary stars. One can readily see that most of member stars fall close to the ZAMS location. There are actually some exceptions (stars \#1, \#3, \#9, \#14, and \#67), which are clearly off (too red or too blue) the MS. We are not going to consider these stars as NGC 1220 likely members.

In conclusion, we would like to argue that the population of stars having $E(B-V)=0.70 \pm 0.15$ mag (26 stars) 
Table 3. Photometry of likely member stars in the field of NGC 1220. In the last column, p.n.m. means probable non member.

\begin{tabular}{|c|c|c|c|c|c|c|c|c|c|}
\hline ID & $X$ (pixel) & $Y($ pixel $)$ & $\bar{V}$ & $(B-V)$ & $(U-B)$ & $Q$ & $E(B-V)$ & Sp.Type & Notes \\
\hline 1 & 967.330 & 15.690 & 14.848 & 0.575 & 0.462 & 0.048 & 0.568 & A1 & p.n.m \\
\hline 3 & 228.090 & 285.270 & 14.480 & 0.709 & 0.526 & 0.016 & 0.713 & B9 & p.n.m. \\
\hline 4 & 567.300 & 397.170 & 13.539 & 0.502 & -0.107 & -0.468 & 0.669 & B7 & \\
\hline 5 & 475.760 & 433.540 & 14.387 & 0.664 & 0.163 & -0.315 & 0.779 & B6 & \\
\hline 6 & 520.020 & 483.510 & 13.923 & 0.579 & -0.049 & -0.466 & 0.745 & A4 & \\
\hline 9 & 492.600 & 9.870 & 17.667 & 0.727 & 0.578 & 0.055 & 0.718 & $\mathrm{~A} 2$ & p.n.m. \\
\hline 13 & 688.280 & 235.520 & 15.743 & 0.656 & 0.524 & 0.052 & 0.648 & $\mathrm{~A} 2$ & \\
\hline 14 & 851.920 & 237.320 & 15.247 & 0.537 & 0.198 & -0.189 & 0.610 & B8 & p.n.m. \\
\hline 15 & 536.950 & 276.220 & 15.442 & 0.537 & 0.150 & -0.237 & 0.626 & B7 & \\
\hline 16 & 864.100 & 327.730 & 16.795 & 0.766 & 0.626 & 0.074 & 0.750 & A3 & \\
\hline 17 & 449.040 & 337.210 & 16.838 & 0.779 & 0.611 & 0.050 & 0.771 & B9 & \\
\hline 23 & 570.490 & 419.710 & 15.627 & 0.623 & 0.319 & -0.130 & 0.676 & B9 & \\
\hline 26 & 550.960 & 473.700 & 15.491 & 0.637 & 0.305 & -0.159 & 0.698 & B9 & \\
\hline 30 & 690.850 & 503.320 & 13.219 & 0.507 & -0.138 & -0.503 & 0.686 & B5 & \\
\hline 35 & 520.440 & 538.900 & 15.966 & 0.737 & 0.519 & -0.012 & 0.750 & B9 & \\
\hline 36 & 373.510 & 541.340 & 16.054 & 0.694 & 0.393 & -0.107 & 0.739 & B9 & \\
\hline 39 & 716.750 & 577.490 & 16.697 & 0.759 & 0.534 & -0.012 & 0.772 & A0 & \\
\hline 48 & 126.610 & 791.540 & 14.783 & 0.608 & -0.017 & -0.455 & 0.770 & B5 & \\
\hline 58 & 246.520 & 214.460 & 16.085 & 0.655 & 0.370 & -0.102 & 0.698 & B9 & \\
\hline 64 & 494.780 & 423.660 & 15.987 & 0.749 & 0.457 & -0.082 & 0.786 & B9 & \\
\hline 66 & 422.610 & 460.180 & 14.023 & 0.627 & -0.024 & -0.475 & 0.796 & B5 & p.n.m. \\
\hline 67 & 440.650 & 525.220 & 14.875 & 0.627 & 0.086 & -0.365 & 0.759 & B5 & \\
\hline 68 & 556.110 & 530.450 & 14.997 & 0.636 & 0.183 & -0.275 & 0.738 & B6 & \\
\hline 69 & 605.160 & 540.530 & 16.387 & 0.700 & 0.475 & -0.029 & 0.719 & A0 & \\
\hline 78 & 537.330 & 350.389 & 17.033 & 0.846 & 0.674 & 0.065 & 0.833 & A3 & \\
\hline 79 & 528.560 & 378.300 & 15.982 & 0.732 & 0.512 & -0.015 & 0.746 & B9 & \\
\hline
\end{tabular}

identifies the brightest members of the open cluster NGC 1220. Later spectral type stars are more difficult to be detected, due to the degeneracy described above. The final photometry of the likely candidate members of NGC 1220 is listed in Table 3, together with individual reddenings and photometric spectral types. The latter have been obtained by deriving intrinsic colours from observed colours and reddenings. We use Johnson (1966) intrinsic colours to infer approximate spectral types. The typical uncertainty in the spectral type is \pm 2 , due to both photometric errors and the difficulty to estimate the luminosity classes.

\subsection{Distance and age}

In Fig. 7 we plot the reddening corrected CMDs for the likely member stars above determined. In both diagrams we have overimposed the empirical Schmidt-Kaler (1982) ZAMS, shifted by $(m-M)_{\mathrm{o}}=11.3 \pm 0.3 \mathrm{mag}$, which provides a nice fit of the stars distribution. This implies that NGC 1220 is located $1800 \pm 200$ pc away from the Sun, where the uncertainty mirrors the difficulty of the fit due to the almost vertical structure of the MS.

From the location of the stars in the $(B-V)$ vs. $(U-B)$ plane, we infer that the stars spectral types range from $B 5$ to $A 5$ by deriving the absolute colours from the ZAMS at the same

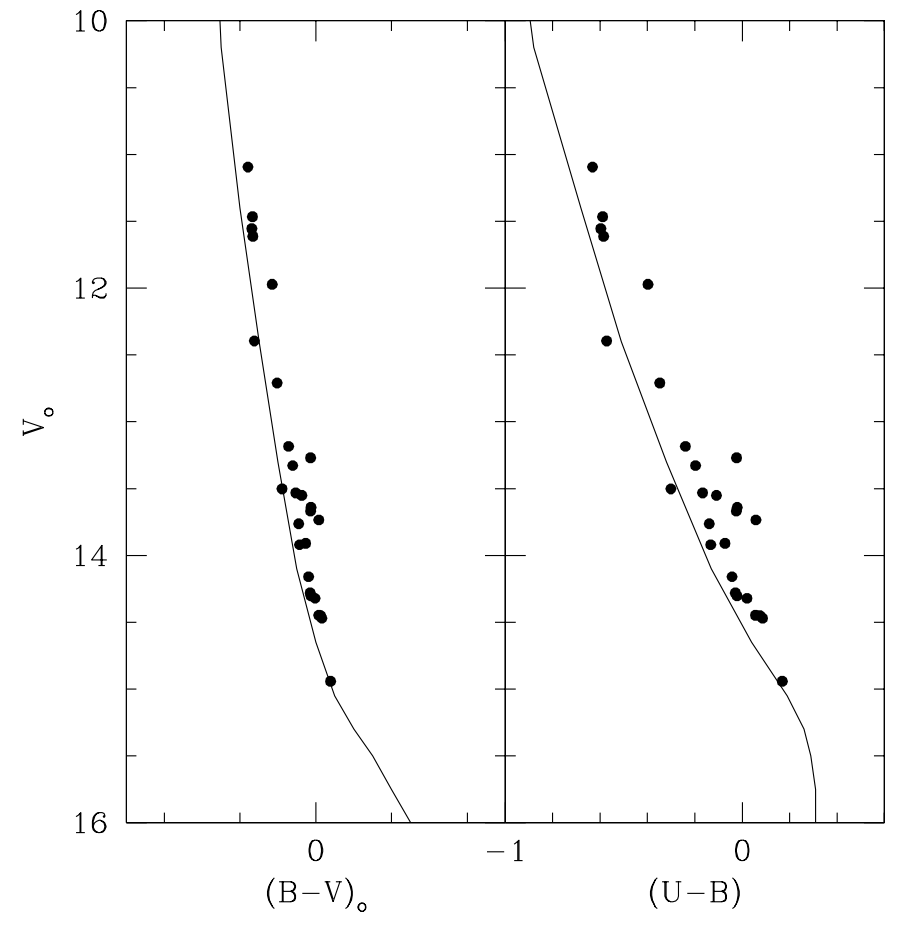

Fig. 7. Reddening corrected CMDs of the likely member stars in the region of NGC 1220. The solid line is an empirical ZAMS shifted by $(m-M)_{V}=13.50 \mathrm{mag}$. 


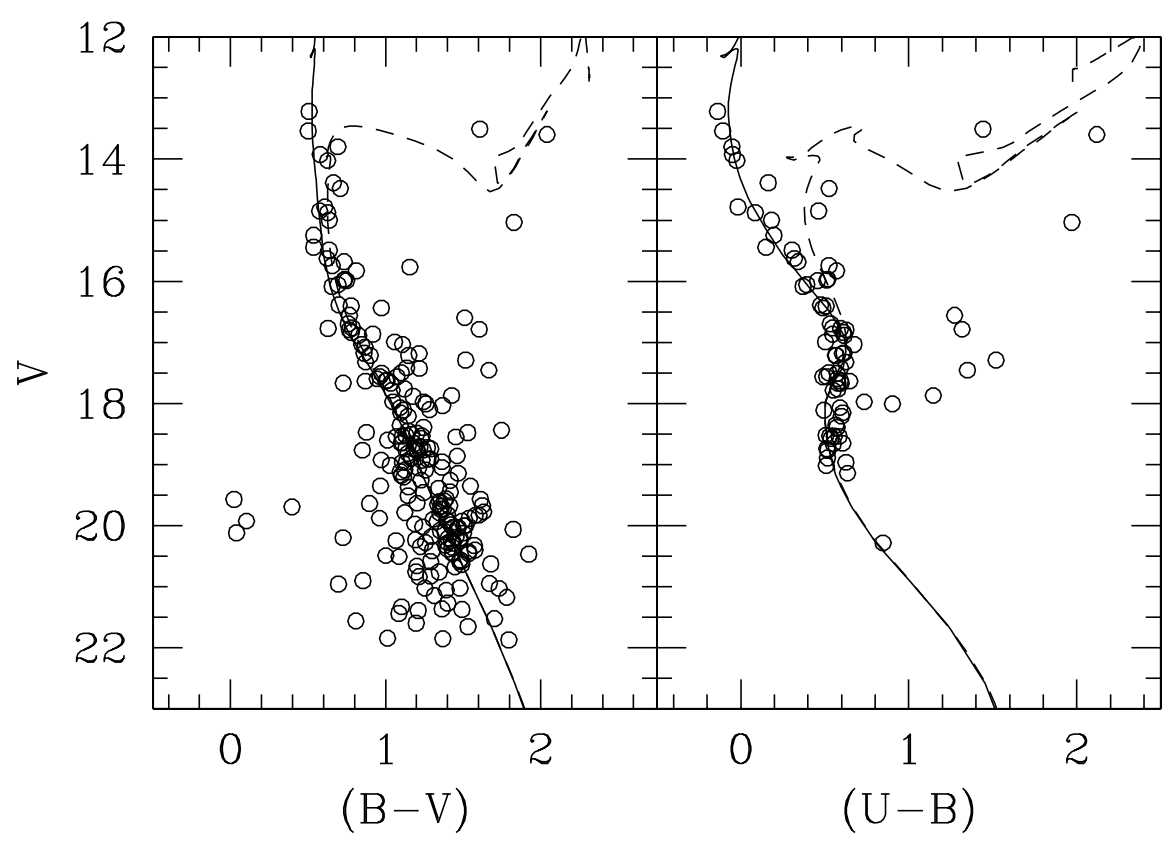

Fig. 8. CMDs of all the stars in the region of NGC 1220. The solid line is a solar metallicity 60 Myr isochrone from Girardi et al. (2000), whereas the dashed one is a 250 Myrs isochrone.

position of the stars (see also Table 3 and previous section). If the stars having $B 5$ spectral type are still along the MS, we derive an age around 50 Myrs for NGC 1220 (Girardi et al. 2000).

We checked this age estimate by fitting CMDs of NGC 1220 with theoretical isochrones from Padova models (Girardi et al. 2000). The result is presented in Fig. 8. In the left panel we plot the NGC 1220 stars in the plane $V$ vs. $(B-V)$, and overimposed two solar metallicity isochrones. The solid line is for the age of $60 \mathrm{Myr}$, the dashed one for the age of $250 \mathrm{Myr}$. This latter has been drawn with the intention to try to fit the three very red stars previously mentioned. The same isochrones have been overlaid to NGC 1220 stars in the $V$ vs. $(U-B)$ diagram (right panel). The overall fit is very good in both diagrams. However, by closely inspecting only the left panel, one could not definitely rule out the larger age for NGC 1220, since the dashed isochrone (250 Myr) nicely fits the bulk of the stars but for only the two bright stars above the TO, which nevertheless might be accounted for by invoking their possible binary nature.

In this respect the CMD in the right panel ( $V$ vs. $(U-B))$ helps a lot in solving the mystery. In fact in this colour combination the colour separation of the bluest stars is much wider. It results very clearly that only the $60 \mathrm{Myr}$ isochrone provides a good fit to the MS stars, thus ensuring us that all the stars redwards the MS are simply field stars. Therefore we conclude that NGC 1220 is a young open cluster, about 60 Myr old.

The Galactocentric coordinates are $X=-9.43(X<0$ means our side of the Galaxy), $Y=1.08$ and $Z=-0.12 \mathrm{kpc}$, considering the distance to the Galactic center to be $R=8 \mathrm{kpc}$ (Reid 1993). The cluster is thus outside the solar circle, in the Perseus arm (Taylor \& Cordes 1993). However, we notice that NGC 1220 is fairly high above the Galactic plane with respect to his age. In fact, it takes at least $10^{7} \mathrm{yr}$ for a young cluster with a typical velocity of $10 \mathrm{~km} \mathrm{~s}^{-1}$ to move about $100 \mathrm{pc}$.

Combining together the estimated age, distance and position in the Galaxy, we conclude that NGC 1220 is a genuine Galactic this disk star cluster, presently located relatively high above the Galactic plane, but presumably formed within the thin disk.

\section{Conclusions}

In this paper we have presented new CCD $U B V$ photometry for the stars in the field of NGC 1220, and provide the first estimates of its fundamental parameters.

Our findings can be summarized as follows:

- NGC 1220 is a compact 20-25 stars group, with a radius of $1.5-2.0$ arcmin, which turns into $0.79-1.05$ pc at the distance of the cluster;

- we identified 26 likely members with spectral type earlier than $A 5$ on the basis of the reddening and the position in the reddening corrected CMDs;

- the cluster turns out to be located about 1800 pc away from the Sun in the Perseus spiral arm;

- we estimate a reddening $E(B-V)=0.70 \pm 0.15$;

- the probable age is around 60 Myrs;

- NGC 1220 is presently located 120 pc above the Galactic plane, relatively high with respect to its age. With the available data it is not possible to conclude whether the cluster formed at some distance above the galactic plane, or formed well within the thin disk and then moved away. This latter hypothesis would imply a non negligible vertical motion of the cluster.

We would finally like to note that more precise estimates of the cluster age can be derived by obtaining spectroscopic classification of the brightest stars. Moreover a proper motion 
study would permit to better distinguish NGC 1220 physical members.

Acknowledgements. This study made use of Simbad and WEBDA catalogs. We acknowledge partial financial support from the brazilian agencies CNPq and Fapesp, and Ministero dell'Università e della Ricerca Scientifica e Tecnologica (MURST) under the program on "Dynamics and Stellar Evolution in Globular Clusters: a Challenge for New Astronomical Instruments".

\section{References}

Carraro, G. 2002a, MNRAS, 331, 785

Carraro, G. 2002b, A\&A, 387, 479

Cudworth, K. M., Martin, S. C., \& DeGioia-Eastwood, K. 1993, AJ, 105,1822
Girardi, L., Bressan, A., Bertelli, \& G., Chiosi, C. 2000, A\&AS, 141, 371

Johnson, H. L. 1966, ARA\&A, 4, 193

Landolt, A. U. 1992, AJ, 104, 340

Lyngå, G. 1987, Catalog of Open Star Cluster Data, Strasbourg, CDS Patat, F., \& Carraro, G. 2001, MNRAS, 325, 1591

Phelps, R. L., Janes, K. A., \& Montgomery, K. A. 1994, AJ, 107, 1079 Reid, M. 1993, ARA\&A, 31, 345

Schmidt-Kaler, Th. 1982, Landolt-Börnstein, Numerical data and Functional Relationships in Science and Technology, New Ser., Group VI, vol. 2(b), ed. K. Schaifers, \& H. H. Voigt (Springer Verlag, Berlin), 14

Taylor, J. H., \& Cordes, J. M. 1993, ApJ, 411, 674

Vazquez, T., Baume, G., Prado, P., \& Feinstein, A. 1996, A\&AS, 166, 75 